\section{Protective effect of different antioxidant agents in UVB-irradiated keratinocytes}

\author{
Sara Salucci, ${ }^{1}$ Sabrina Burattini, ${ }^{1}$ \\ Francesca Buontempo, ${ }^{2}$ \\ Alberto Maria Martelli, ${ }^{2}$ \\ Elisabetta Falcieri, ${ }^{1}$ Michela Battistelli ${ }^{1}$ \\ ${ }^{1}$ Department of Biomolecular Sciences, \\ University of Urbino "Carlo Bo" \\ ${ }^{2}$ Department of Biomedical and \\ Neuromotor Sciences, University of \\ Bologna, Italy
}

\section{Abstract}

Skin cells can respond to UVB-induced damage either by tolerating it, or restoring it through antioxidant activation and DNA repair mechanisms or, ultimately, undergoing programmed cell death, when damage is massive. Nutritional factors, in particular, food antioxidants, have attracted much interest because of their potential use in new preventive, protective, and therapeutic strategies for chronic degenerative diseases, including skin inflammation and cancer. Some polyphenols, present in virgin olive oil, well tolerated by organism after oral administration, show a variety of pharmacological and clinical benefits such as antioxidant, anti-cancer, anti-inflammatory, and neuro-protective activities. Here, the protective effects of antioxidant compounds against UV-induced apoptosis have been described in HaCaT cell line. Human keratinocytes were pre-treated with antioxidants before UVB exposure and their effects have been evaluated by means of ultrastructural analyses. After UVB radiation, a known cell death trigger, typical apoptotic features, absent in control condition and in antioxidant alone-treated cells, appear. An evident numerical decrease of ultrastructural apoptotic patterns and TUNEL positive nuclei can be observed when natural antioxidants were supplied before cell death induction. These data have been confirmed by molecular investigation of caspase activity. In conclusion, this paper highlights antioxidant compound ability to prevent apoptotic cell death in human keratinocytes exposed to UVB, suggesting, for these molecules, a potential role in preventing skin damage.

\section{Introduction}

The skin is the largest organ of the body, comprises a surface area of approximately $1.5-2.0 \mathrm{~m}^{2}$ and protects the underlying tissues through melanogenesis, by acting as an effective barrier against the detrimental effects of environmental, chemical and physical agents. ${ }^{1}$

Exposure to solar UV radiation is considered a major source of damage for human skin, it induces several harmful responses, including erythema, immunosuppression, edema, sunburn, keratinocyte hyperplasia, wrinkling, dryness, mottled pigment abnormalities, premature aging and skin cancer. ${ }^{2,3}$

UV radiation (100-400 $\mathrm{nm}$ wavelength) is composed of UVA (320-400 nm), UVB $(280-320 \mathrm{~nm})$ and UVC (200-280 $\mathrm{nm})$ rays. UVB radiation represents the major cause of serious skin disorders and genotoxic damage. ${ }^{4,5}$ Exposure of mammalian skin to UV light impairs antioxidant defense mechanisms and increases the cellular levels of reactive oxygen species (ROS), which, in turn, damage lipids, proteins and nucleic acids, thus contributing to the process of photocarcinogenesis and photoaging. ${ }^{6,7}$ In addition, exposure of cells to UVB radiation results in the loss of keratinocyte viability, an increase in membrane blebbing, cytoskeletal molecular changes, and apoptosis. ${ }^{8}$ Apoptosis is a well defined, highly regulated form of programmed cell death that is evident in normal skin (e.g., during keratinocyte differentiation) as well as in pathological conditions. UVB-induced apoptosis is mediated by apoptotic signals, activating two main pathways: intrinsic (mitochondrial) and extrinsic (death receptor-dependent). ${ }^{9}$

This paper discusses the effect of wellknown antioxidant molecules against UVB radiation in a keratinocyte cell line (HaCaT). The compounds, chosen for their ROS scavenger and anti-apoptotic properties in keratinocytes, are Melatonin (Mel), Creatine (Cr), Hydroxytyrosol (HyT), Tyrosol (TyR) and, for the first time, Hydroxytyrosyl Laurate (Laur-HyT) and Hydroxytyrosyl Myristate (Myr-HyT). ${ }^{10-14}$

$\mathrm{Mel}$, a pineal hormone derived from tryptophan metabolism, is known for its antioxidant and anti-apoptotic action against UVB damage. ${ }^{11,14}$ It was originally identified as the main secretory product of the pineal gland, later demonstrated to be synthesized in a variety of extrapineal sites, with bioactivity $v s$ a number of targets in single cells, animals and humans. ${ }^{15} \mathrm{Mel}$ can act as a receptor-independent antioxidant, ${ }^{16}$ as an antiaging substance and as an anticar-
Correspondence: Michela Battistelli, Department of Biomolecular Sciences, University of Urbino "Carlo Bo", Via Ca' le Suore 2, 61029 Urbino (PU), Italy.

Tel.: +39.0722.304269

E-mail: michela.battistelli@uniurb.it

Key words: Keratinocytes; UVB radiation; natural antioxidants; ultrastructure; TUNEL.

Acknowledgements: this work was supported by the University of Urbino "Carlo Bo", in particular by the "Progetto di Valorizzazione DISB 2017". We would like to thank Dr. Francesco M. Giordano and Dr. Debora Burini for their skillful technical assistance.

Received for publication: 8 March 2017

Accepted for publication: 28 July 2017.

This work is licensed under a Creative Commons Attribution-NonCommercial 4.0 International License (CC BY-NC 4.0).

(C) Copyright S. Salucci et al., 2017

Licensee PAGEPress, Italy

European Journal of Histochemistry 2017; 61:2784 doi:10.4081/ejh.2017.2784

cinogenic factor within a wide range of concentrations. ${ }^{17}$

$\mathrm{Cr}$ is a molecule with protective effects in neurological and atherosclerotic diseases, in brain and nerve traumatic injury and in UV-induced mitochondrial DNA mutations in skin cells ${ }^{18}$ and in skeletal muscle cells after $\mathrm{H}_{2} \mathrm{O}_{2}$ exposure. ${ }^{19}$

HyT, a natural antioxidant polypheno ${ }^{13}$ present in virgin oil, shows a variety of pharmacological effects, such as antioxidant, anti-cancer, anti-inflammatory and neuro-protective activities..$^{20}$

HyT, obtained by chemical or enzymatic hydrolysis of oleuropein glucoside, or by chemical synthesis, is the main biophenol in olive oil, regularly assimilated in humans.

TyR, a liposoluble, non-carboxyl monophenol, is one of the most representative phenols in olive leaves and in extra-virgin olive oil. Its antioxidant and anti-proliferative activities, as well as its ability to inhibit pro-oxidation processes on human LDL particles, ${ }^{21}$ have been demonstrated.

Because of the limited solubility of HyT and TyR in lipid media, the search for new lipophilic derivatives with enhanced properties is of great interest. Several authors modified the chemical structure of these compounds increasing the alkyl chain and obtaining the ester or eter derivatives. Mateos et al. and Madrona et al. ${ }^{22,23}$ demonstrated that HyT derivatives with short and medium acyl side chains, such as the acetyl, butyryl and lauryl, are much more effective 
in protecting the $\mathrm{RBCs}$ against $\mathrm{AAPH}$ induced hemolysis than HyT. The greatest efficiency was obtained when the alkyl chain has a length around $12 \mathrm{C}$-atoms (Laur-HyT). Moreover, Burattini et al. ${ }^{13}$ demonstrated that Laur-HyT has a protective antioxidant effect against $\mathrm{H}_{2} \mathrm{O}_{2}$ treatment, having a role in the prevention of apoptotic death in normal and tumor cells.

For that, in this work, Laur-HyT (12 Catoms) and Myr-HyT (14 C-atoms) activities against UVB rays, have been analyzed to understand if the antioxidant effect also occurs in the presence of different number and position of the phenolic hydroxyl groups.
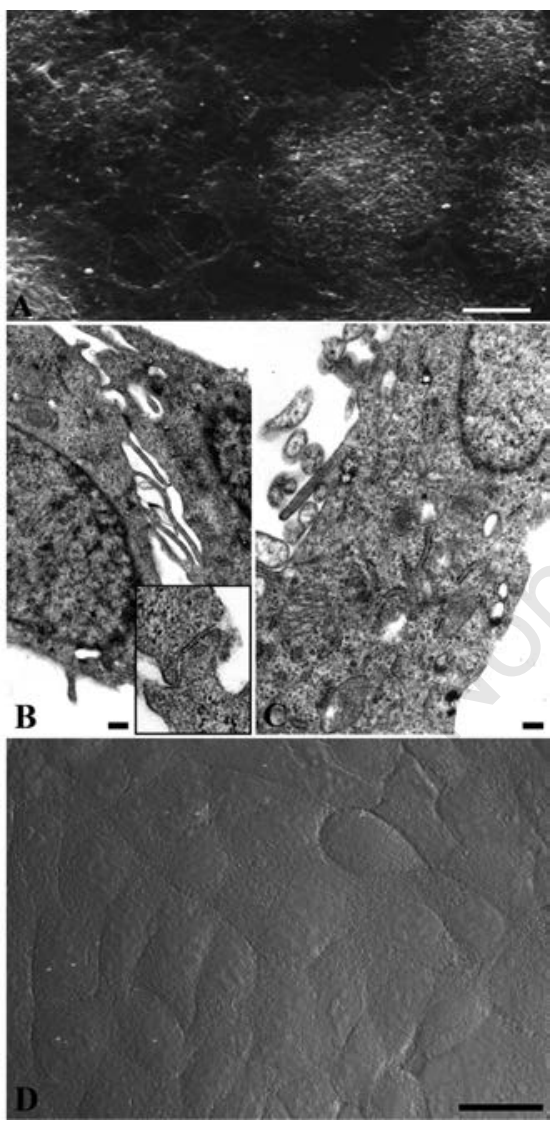

Figure 1. Control cells observed at SEM (A), TEM (B) and CLSM (C). Cells appear mostly polygonal, flattened $(A, B)$ with preserved RER and mitochondria $(C)$ and frequent close cell contacts ( $B$, inset). After TUNEL reaction no labelled nuclei can be observed (D). Scale bars: A) 10 um; B,C) $0.2 \mu \mathrm{m}$; inset in B) $0.1 \mu \mathrm{m}$; D) $25 \mu \mathrm{m}$.

\section{Materials and Methods}

\section{Cell culture}

HaCaT cells, spontaneously immortalized human keratinocytes, were grown in DMEM supplemented with $10 \%$ heat-inactivated fetal bovine serum, $2 \mathrm{mM}$ glutamine and $1 \%$ antibiotics and were maintained at $37^{\circ} \mathrm{C}$ in humidified air with $5 \% \mathrm{CO}_{2}$. Cell behavior was progressively monitored by means of inverted microscope.

\section{Apoptosis induction and antioxidant administration}

For apoptosis induction cells were cultured to $80 \%$ confluence and washed with PBS, covered with a thin layer of PBS and exposed to UVB for $10 \mathrm{~min}$. The irradiating source consisted of a lamp generating UVB light in the range of 290-320 nm with an emission peak at $312 \mathrm{~nm}$ (Transilluminator 2000, Bio-Rad Laboratories, Hercules, CA, USA). After the addition of fresh medium, cells were further incubated for $2 \mathrm{~h}$.

Different aliquots of $\mathrm{HaCaT}$ cells were treated for $24 \mathrm{~h}$ with $100 \mu \mathrm{M}$ Mel, or $30 \mu \mathrm{M}$ $\mathrm{Cr}$ monohydrate, or $20 \mu \mathrm{M}$ HyT, or $5 \mu \mathrm{M}$ TyR, or $5 \mu \mathrm{M}$ Laur-HyT, or $5 \mu \mathrm{M}$ Myr-HyT before UVB exposure. Antioxidant concentrations have been chosen since they did not affect $\mathrm{HaCaT}$ cell viability and proliferation as demonstrated in our previous works. ${ }^{14,24}$

Mel (Sigma-Aldrich, St. Louis, MO, USA) was first dissolved in absolute ethanol at the initial concentration of 100 $\mathrm{mM}$, and then diluted at final $100 \mu \mathrm{M}$ concentration in culture medium. Cr (SigmaAldrich) was dissolved in complete culture medium at final $3 \mathrm{mM}$ concentration. HyT and TyR were recovered with $95 \%$ purity from olive oil wastewaters, and further purified by column chromatography. HyT ester derivatives (Laur-HT and Myr-HyT) were prepared according to Mateos et al..$^{22}$ For all treatments, morpho-functional analyses have been performed after $2 \mathrm{~h}$ post incubation, which follows UVB radiation.

\section{Scanning electron microscopy (SEM)}

Control and treated cells were directly processed on coverslips in Petri dishes.

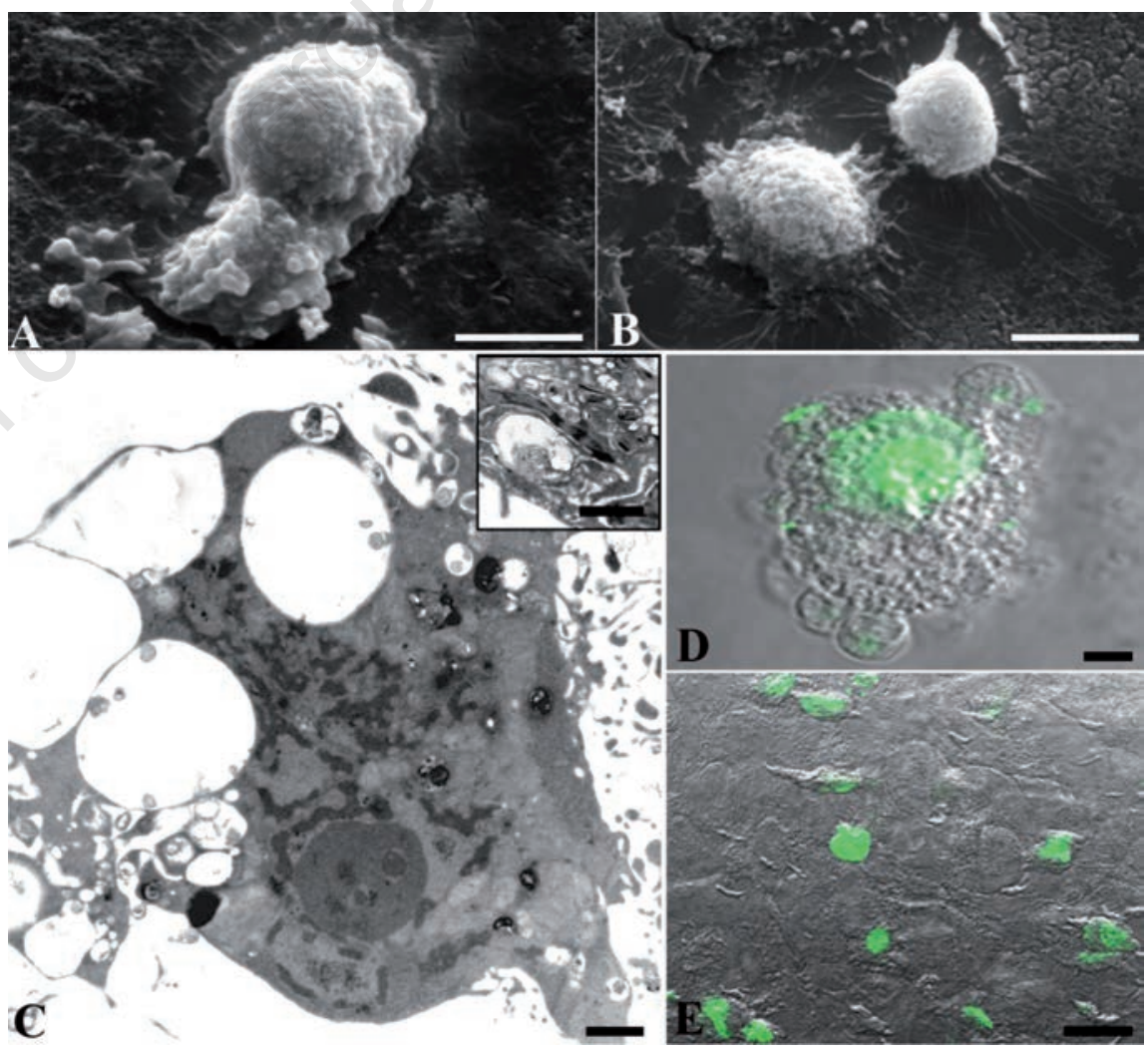

Figure 2. HaCaT cells exposed to UVB and observed at SEM (A,B), TEM (C) and CLSM (D). Apoptotic features, as well as a diffuse positivity to TUNEL reaction, appear. Autophagic vacuoles occasionally appear at TEM observation (C, inset). Scale bars: A,B) $10 \mu \mathrm{m}$; $C$ and inset) $1 \mu \mathrm{m}$; D) $5 \mu \mathrm{m}$; E) $25 \mu \mathrm{m}$. 
After careful washing, monolayers were fixed with $2.5 \%$ glutaraldehyde in $0.1 \mathrm{M}$ phosphate buffer for $1 \mathrm{~h}$. All the specimens were post-fixed with $1 \% \mathrm{OsO}_{4}$ in the same buffer for $1 \mathrm{~h}$. After alcohol dehydration, samples were critical point dried, gold sputtered and observed with a scanning electron microscope. ${ }^{25}$

\section{Transmission electron microscopy (TEM)}

HaCaT cells were washed, immediately fixed in situ with $2.5 \%$ glutaraldeyde in $0.1 \mathrm{M}$ phosphate buffer for $15 \mathrm{~min}$, gently scraped and centrifuged at $1200 \mathrm{rpm}$. The pellets were fixed in $2.5 \%$ glutaraldehyde for additional $30 \mathrm{~min}$.

Cells were post-fixed in $1 \% \mathrm{OsO}_{4}$ for $1 \mathrm{~h}$, alcohol dehydrated and embedded in araldite. ${ }^{26,27}$ Thin sections were stained with uranyl acetate and lead citrate and analyzed at transmission electron microscope.

\section{TUNEL}

Control and treated cells, directly processed on coverslips in Petri dishes, were washed and fixed with $4 \%$ paraformaldehyde in PBS, $\mathrm{pH}$ 7.4, for 30 min, rinsed with PBS and permeabilized with a 2:1 mixture of ethanol and acetic acid for $5 \mathrm{~min}$ at $-20^{\circ} \mathrm{C}$. For the TUNEL technique, all reagents were part of the ApopTag Plus $^{\circledR}$ kit (D.B.A., Dallas, TX, USA) and the procedures were carried out according to the manufacturer's instructions, as described by Salucci et al. ${ }^{14}$ Specimens were observed with a Leica TCS-SP5 connected to a DMI 6000 CS Inverted Microscope (Leica Microsystems CMS $\mathrm{GmbH}$, Mannheim, Germany); excitation was at $488 \mathrm{~nm}$ and emission signals were detected at $517 \mathrm{~nm}$.

\section{Trypan blue (TB) exclusion assay}

TB exclusion assay was performed to identify living cells and quantify dead cell number percentage in all experimental conditions. Differences in the percentages of viable cells among groups were determined using one-way analysis of variance (ANOVA), followed by Tukey HSD post-hoc tests to evaluate individual group differences. Significance was set at $\mathrm{P}<0.05$. Data were collected from three independent experiments.

\section{Western blotting}

Protein assay was performed using the Bio-Rad Protein Assay according to the manufacturer's instructions. Cells were lysed at $10^{7} / \mathrm{mL}$ in RIPA lysis buffer (Thermo Scientific, Waltham, MA, USA). Lysates were sonicated to shear DNA and

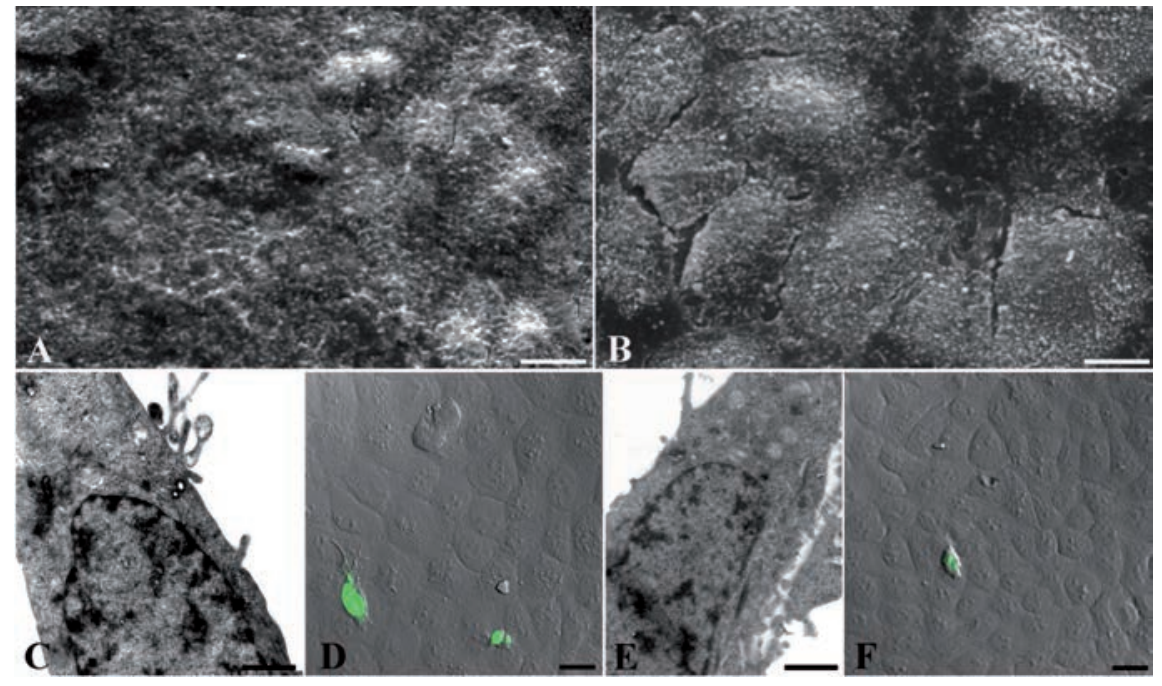

Figure 3. Mel (A,C,D) and $\mathrm{Cr}(\mathrm{B}, \mathrm{E}, \mathrm{F})$ treated cells before UVB exposure and observed at SEM (A,B), TEM (C,E) and CLSM (D,F). All compounds induce an evident apoptotic pattern numerical decrease. Scale bars: A,B) $10 \mu \mathrm{m}$; C,E) $0.5 \mu \mathrm{m}$; D,F) $25 \mu \mathrm{m}$.
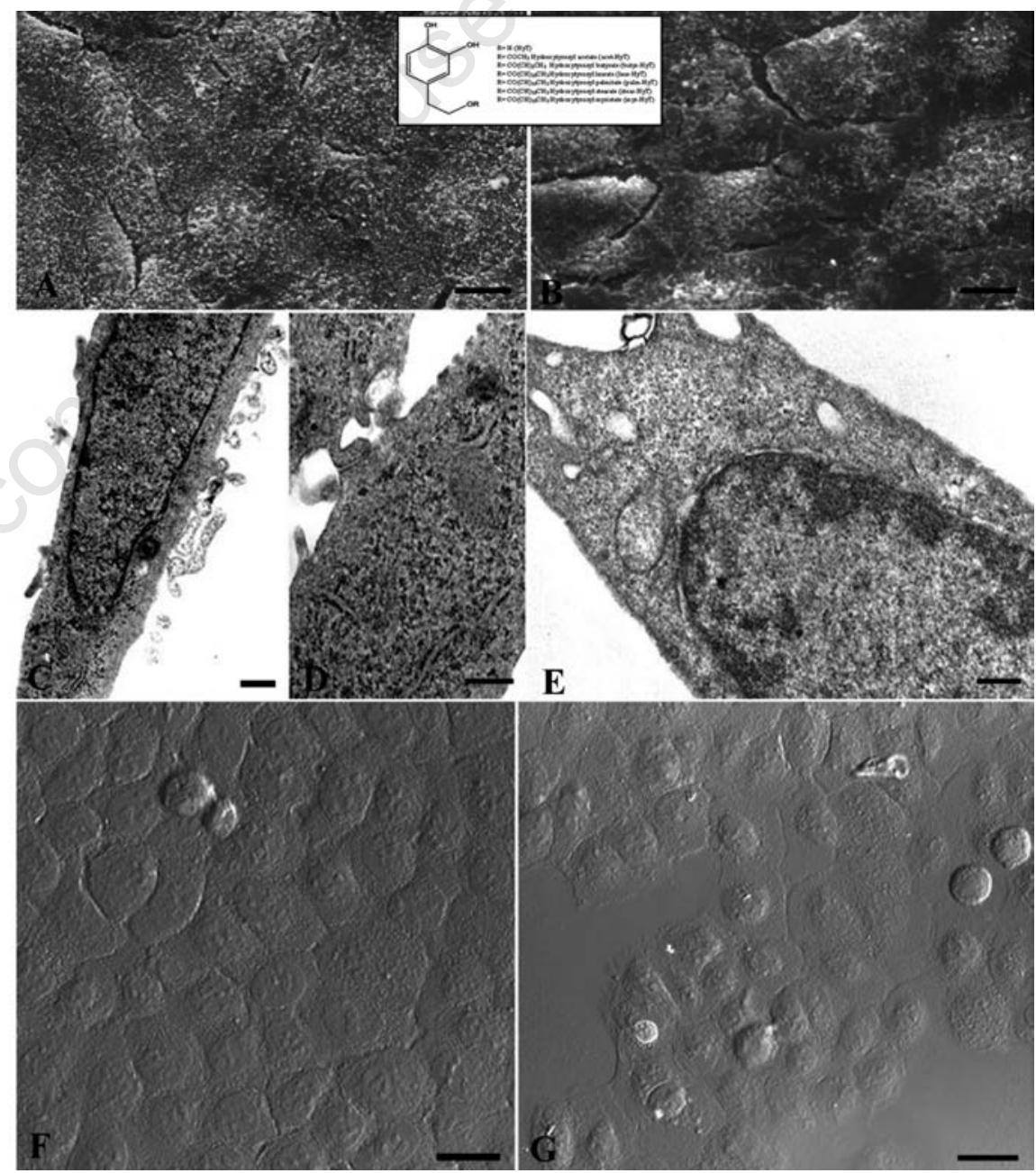

Figure 4. HyT (A,C,D,F), TyR (B,E,G) treated cells before UVB exposure and observed at SEM (A,B), TEM (C,D,E) and CLSM (F,G). Scale bars: A,B) $10 \mu \mathrm{m}$; C) $0.5 \mu \mathrm{m} ; \mathrm{D}, \mathrm{E}) 0.25$ $\mu \mathrm{m} ; \mathrm{F}, \mathrm{G}) 25 \mu \mathrm{m}$. 
boiled for $5 \mathrm{~min}$ with reducing sample buffer. Protein separated $(25 \mu \mathrm{g})$ on sodium dodecylsulphate polyacrylamide gel electrophoresis (SDS-PAGE) was transferred to nitrocellulose membranes using a semidry blotting apparatus. Membranes were saturated for $60 \mathrm{~min}$ at room temperature in blocking buffer, then incubated overnight at $4^{\circ} \mathrm{C}$ with primary antibody for caspase-8, $-9,-3$ and PARP (Cell Signaling Technology, Danvers, MA, USA). After washing in PBS containing $0.1 \%$ Tween 20 , samples were incubated for $1 \mathrm{~h}$ at room temperature with peroxidase-conjugated secondary antibody diluted 1:2000 in PBSTween 20, and washed as above. ${ }^{24}$ Bands were visualized by the ECL method and images were achieved through ChemiDocit2 Imager (UVP, Upland, CA, USA). Densitometric analysis of cleaved caspase western blotting bands has been performed. Data are expressed as ratio between mean optical density (OD) of cleaved caspase band and mean OD of actin band (protein control), in arbitrary units.

\section{Results}

Cells treated with Mel, Cr, HyT, Tyr, Laur-HyT and Myr-HyT alone appeared very similar to control ones, according to quantitative analyses previously reported, ${ }^{10-14,17,19}$ indicating that these compounds did not affect cell viability and/or proliferation. Moreover, TUNEL reaction appeared negative in all conditions (data not shown). ${ }^{28}$

\section{HaCaT cells exposed to UVB radia- tion}

SEM and TEM observations showed how keratinocytes appeared in control condition (Figure 1). Cells were mostly polygonal, flattened and closely confluent (Figure 1A), and displayed a large nucleus with diffuse chromatin (Figure 1B). Close cell contacts (Figure 1B, inset) frequently appeared, as expected for epidermal origin cells. In the cytoplasm well preserved RER and mitochondria (Figure 1 B,C) could be observed. No DNA fragmentation could be revealed in control condition, as confirmed by TUNEL reaction negativity (Figure 1D).

Differently, UVB irradiated samples revealed deep changes in monolayer organization, as well as significant surface and inner cell ultrastructural alterations (Figure 2). Cell monolayer showed a significant decrease in cell confluence, with cell detachment and the appearance of empty spaces, correlated to cell rounding and blebbing (Figure 2 A,B). Apoptotic patterns, such as chromatin margination and condensation, as well as diffuse cytoplasmic vacuolization, have been detected (Figure 2C). TEM analysis also revealed numerous autophagic vacuoles (Figure $2 \mathrm{C}$, inset). In addition, after UVB radiation exposure, several TUNEL positive, strongly fluorescent nuclei have been detected (Figure 2F). In Figure 2D, a higher magnification of a single cell could be observed where it is possible visualized membrane blebs and DNA cleavage. As also quantified through TB assay, a massive damage appeared after UVB exposure, generating about $60 \%$ dead cells if compared to control condition.

\section{Mel and $\mathrm{Cr}$ action}

Mel (Figure 3 A,C,D) or Cr (Figure 3 B,E,F) treatments, before cell death induction, caused an evident attenuation of apoptotic patterns. A better morphology was observed in all experimental conditions and
TUNEL positive nuclei underwent an evident numerical decrease (Figure $3 \mathrm{D}, \mathrm{F}$ ). After $\mathrm{Mel}$ and $\mathrm{Cr}$ pre-treatment cells appeared similar to the control ones. SEM observations showed polygonal, flattened and closely confluent cells (Figure 3 A,B). At TEM, well preserved RER and mitochondria could be evidenced. Nuclear chromatin appeared diffuse both in $\mathrm{Mel}$ and in $\mathrm{Cr}$ pre-treated cells (Figure $3 \mathrm{C}, \mathrm{E}$ ). The effects of pre-treatment with $\mathrm{Mel}$ and $\mathrm{Cr}$, known in the literature on various cell lines, can be so confirmed in $\mathrm{HaCaT}$ cells too and have been considered by Authors a control condition.

\section{Hyt, Tyr and ester derivative action}

Also with Hyt (Figure 4 A,C,D,F) or Tyr (Figure 4 B,E,G) pre-treatment, a good preservation of cell morphology could be observed: cells appeared polygonal, flattened and confluent at SEM observation
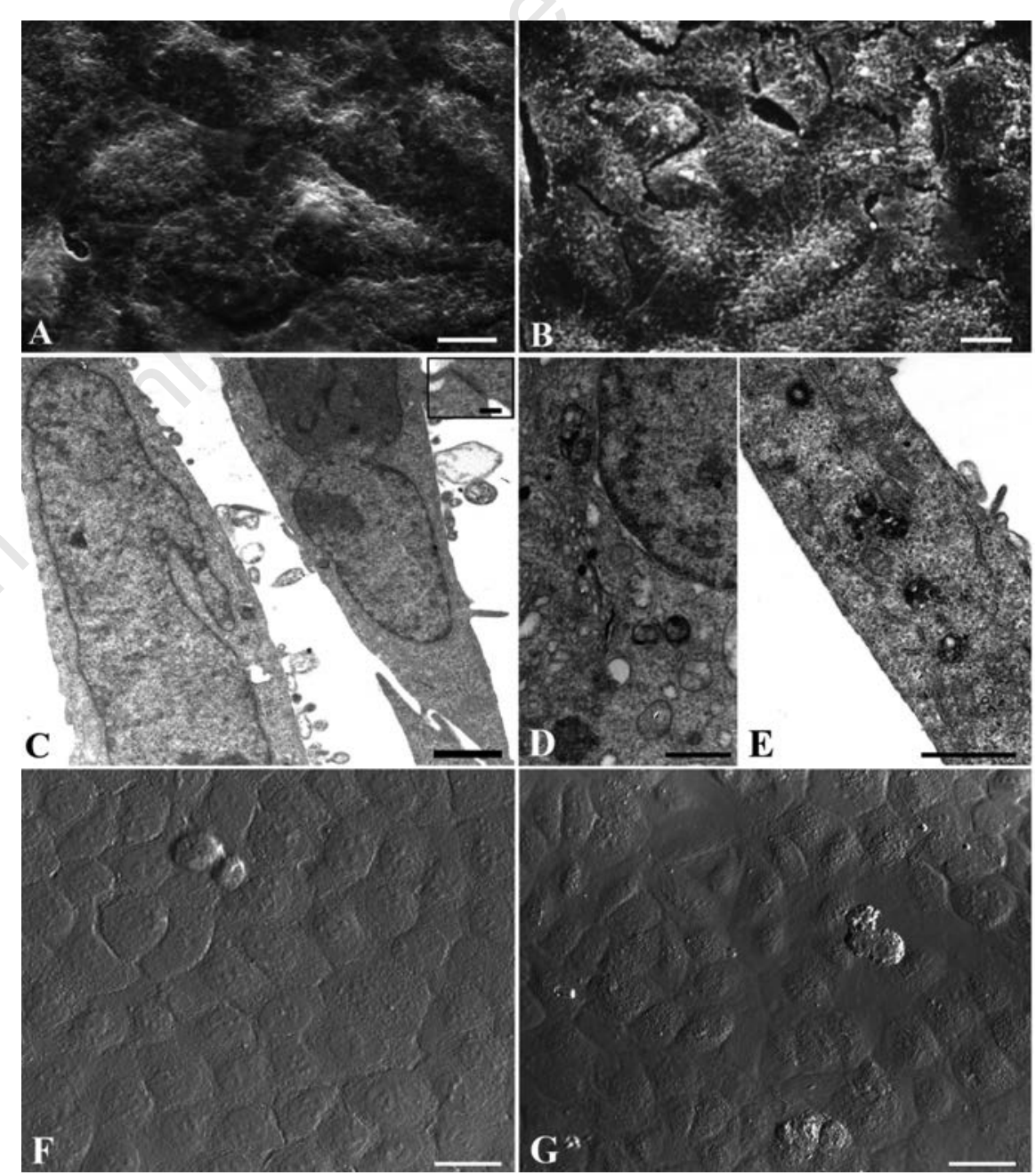

Figure 5. Laur-HyT (A,C,F), Myr-HyT (B,D,E,G) treated cells before UVB exposure and observed at SEM (A,B), TEM (C,D,E) and CLSM (F,G). Scale bars: A,B) $10 \mu \mathrm{m}$; C,D,E) $1 \mu \mathrm{m}$; F,G) $25 \mu \mathrm{m}$; inset of C) $0.1 \mu \mathrm{m}$. 
(Figure 4 A,B). The cytoplasmic organelles were preserved (Figure 4 C,E) and intracellular junction could be appreciated (Figure 4D). Moreover, few positive TUNEL nuclei appeared after Hyt (Figure 4F) and Tyr (Figure 4G) pre-treatment. Even pre-treatment with Laur-HyT (Figure 5 A,C,F) and Myr-HyT (Figure 5 B,D,E,G) produced good results, at SEM observation we have seen the typical morphology of control cells. At TEM close cell contacts (inset in Figure 5C) could be frequently observed. In the cytoplasm we observed a well preserved RER and mitochondria (Figure $5 \mathrm{C}$ ).

\section{TB assay and molecular analyses}

Antioxidant protection has been confirmed and quantified by TB assay (Figure 6) evidencing the ability of these compounds to counteract cell death induced by UVB radiation, sensibly decreasing dead cell number. In particular, oil polyphenols appear the better candidates to prevent UVB damage. A well preserved cellular condition in control samples and a cellular viability decrease after UVB exposure appeared. Interestingly, the antioxidant pretreatments were able to significantly reduce cell death $(\mathrm{P}<0.01)$. In $\mathrm{Cr}$ pre-treated samples the dead cell number reduction was significant too, but to a lesser extent $(\mathrm{P}<0.05)$. To evaluate anti-apoptotic action of all compounds against UVB radiation, caspase involvement has been evaluated though western blotting analysis. Densitometric analyses showed that caspase-8, -9, -3 and PARP activation, absent in control condition, could be observed after UVB exposure while it was reduced by the different pre-treatments. In Figure 6, the densitometric analyses demonstrated the ability of antioxidants to reduce caspase activation which appeared up-regulated after UVB exposure.

\section{Discussion}

This work demonstrates that $\mathrm{Mel}, \mathrm{Cr}$, HyT, Tyr, Laur-HyT, and Myr-HyT are able to prevent UVB-induced apoptosis in a keratinocyte cell line. A massive DNA damage can be inhibited by antioxidant administration, able to significantly reduce cell death, to prevent ultrastructural apoptotic patterns appearance as well as DNA fragmentation, and to reduce caspase activation. All scavengers used in this work, with particular attention to Mel, HyT and Tyr which showed the major anti-apoptotic properties, could represent potential tools in the treatment of UVB exposure-induced skin disorders. As demonstrated in this work, TUNEL reaction evidenced in human keratinocytes after UVB radiation the presence of in situ DNA fragmentation, a typical apoptotic feature. All pre-treatments are able to reduce DNA cleavage and, as consequence, to prevent apoptotic cell death.

In particular, we demonstrate that Mel, which has several functions also in cutaneous biology, ${ }^{29-32}$ reduces apoptotic features in an experimental skin model. This behavior is in accordance with previous studies, which had shown the down-regula-

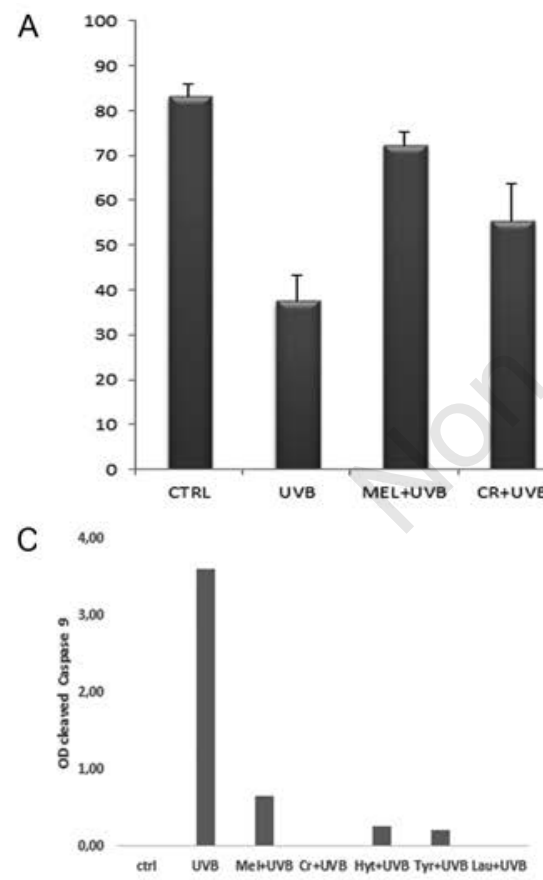

B

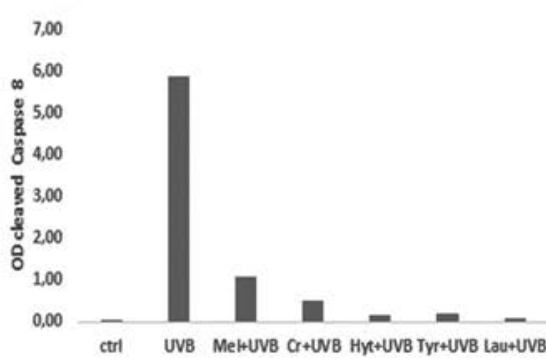

E

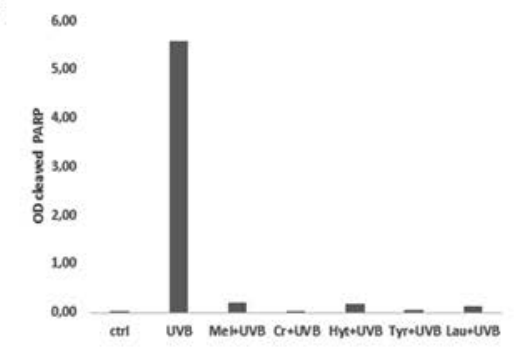

Figure 6. TB assay (A): percentage analysis of viable cells for each treatment. All pre-treatments reduced dead cell number in a significant way. Data are from three separate experiments and are presented as mean \pm standard deviation. Significance has been calculated through ANOVA test $(\mathrm{F}=\mathbf{2 4 . 6 4}$ with $\mathrm{P}<\mathbf{0 . 0 1})$ and Tukey HSD; ${ }^{* *} \mathrm{P}<0.01$ UVB $v$ s CTRL, MEL, HYT, L-HT, TYR, MYR; ${ }^{*} \mathrm{P}<0.05$ UVB $v s \mathrm{CR}$. Densitometric analysis of cleaved caspases-8, -9, $-3(\mathrm{~B}, \mathrm{C}, \mathrm{D})$ and $\mathrm{PARP}(\mathrm{E})$ western blotting bands. Histograms evidenced no activation in untreated cells (ctrl). Caspases and PARP activation could be observed after UVB exposure while it was reduced by the different pre-treatments. Data were representative of three independent experiments performed in triplicate and SD was not shown because less than $10 \%$. Data were expressed as ratio between mean optical density (OD) of cleaved caspase band and mean OD of actin band (protein control), in arbitrary units. 
tion of mitochondrial cytochrome c release and the inhibition of caspase- 9 and -3 activation in Mel treated samples. ${ }^{33,34}$ In addition, interestingly, human skin is able to produce Mel and its metabolites, with strong antioxidant activity ${ }^{35,36}$ exerting intriguingly inhibitory effects in melanoma cells $^{37}$ and suppressing activity of UVBinduced ROS formation. ${ }^{38}$

On the other hand, Cr effect vs UVB radiation has been also demonstrated in keratinocytes in vitro. In particular, apoptotic signs, DNA fragmentation and caspase activation decrease, even if with a lesser extent, in Cr-treated samples before UVB exposure. Despite the important function of $\mathrm{Cr}$ as an essential energy precursor, its endogenous synthesis in humans, sometimes, is not sufficient and it has to be supplemented by daily food intake. Moreover, cutaneous cells may show a Cr level decrease, probably caused by stress or age-related dermal vascularization decline. ${ }^{39}$ Thus, these findings suggest that $\mathrm{Cr}$ supplementation could result in a lower UVB-induced DNA damage in skin cells.

Finally, HyT and TyR, natural dietary polyphenols, well absorbed in the gastrointestinal tract, show anti-apoptotic effects against UVB radiation in $\mathrm{HaCaT}$ cells. HyT and TyR can be considered potential candidates to modify cellular response to UVB radiation. Moreover, these findings demonstrate that synthetic lipophilic hydroxytyrosyl esters can be considered anti apoptotic molecules able to significantly counteract UVB-induce damage, but with a lesser extent respect to the HyT. UVB radiation causes serious injury to skin cells and tissues (and especially to the epidermis) by inducing the production of inflammatory mediators and DNA lesions and, ultimately, by provoking cellular apoptosis.

In this work, the effect of different antioxidant compounds, chosen for their known antioxidant powers in different cell models, has been analyzed in human keratinocytes in vitro. These findings demonstrate that all compounds can be considered as anti-apoptotic agents against UVBinduced apoptosis. Antioxidants appear able to counteract the appearance of apoptotic ultrastructural features, such as chromatin condensation, cytoplasmic vacuolization and in situ DNA fragmentation, which clearly appear in cells exposed to UVB alone. Moreover, molecular analyses demonstrated the capacity of these compounds to prevent, at least in part, caspase activation, which were upregulated after UVB treatment alone.

This work also enhanced the role of oil polyphenol in preventing UVB apoptotic cell death, evidencing the potential antiapoptitc effect exerted by HyT or TyR, the main components of the phenolic fraction of virgin olive oil. Moreover, the effect of HyT esters of $\mathrm{C} 12$ or $\mathrm{C} 14$ fatty acids, with increased lipophilicity, has been evaluated to understand if the antiapoptotic effect also occurs in the presence of different number and position of the phenolic hydroxyl groups. Hyt derivatives with medium acyl side, such as lauryl or myristyl, are less efficient to protect keratinocytes against UVB than HYT. However, they are able to significantly counteract cell death and thank to their chemical structure, they penetrate, better than HyT, through the human corneum stratum and viable epidermis membranes as described by Procopio et al. ${ }^{40}$

In conclusion, considered the general need for safe and effective skin protection against UVB-induced redox alterations, these compounds, able to reduce in vitro UVB-induced keratinocyte apoptosis, could represent important sun damage-preventing molecules, potentially useful in vivo, too.

\section{References}

1. Nichols JA, Katiyar SK. Skin photoprotection by natural polyphenols: antiinflammatory, antioxidant and DNA repair mechanisms. Arch Dermatol Res 2010; 302:71-83.

2. Lahtz C, Kim SI, Bates SE, Li AX, Wu X, Pfeifer GP. UVB irradiation does not directly induce detectable changes of DNA methylation in human keratinocytes [version 1; referees: 2 approved]. F1000 Res 2013;2:45.

3. Strozyk E, Kulms D. The role of AKT/mTOR pathway in stress response to UV-irradiation: implication in skin carcinogenesis by regulation of apoptosis, autophagy and senescence. Int $\mathrm{J}$ Mol Sci 2013;14:15260-85.

4. Gallagher RP, Lee TK, Bajdik CD, Borugian M. Ultraviolet radiation. Chronic Dis Can 2010;29:51-68.

5. Huynh TT, Chan KS, Piva TJ. Effect of ultraviolet radiation on the expression of pp38MAPK and furin in human keratinocyte-derived cell lines. Photodermatol Photoimmunol Photomed 2009;25:20-9.

6. Marrot L, Meunier JR. Skin DNA photodamage and itsbiological 482 consequences. J Am Acad Dermatol 2008;58: 139-48.

7. Pillai S, Oresajo C, Hayward J. Ultraviolet radiation and skin aging: roles of reactive oxygen species, inflammation andprotease activation, and strategies for prevention ofinflammation-induced matrix degradation - a review. Int J Cosmet Sci 2008;27:17-34.

8. Park G, Kim HG, Hong SP, Kim SY, Oh MS. Walnuts (seeds of Juglandis sinensis L) protect human epidermal keratinocytes against UVB-induced mitochondria-mediated apoptosis through upregulation of ROS elimination pathways. Skin Pharmacol Physiol 2014;27:132-40.

9. Van Laethem A, Garmyn M, Agostinis P. Starting and propagating apoptotic signals in UVB irradiated keratinocytes. Photochem Photobiol Sci 2009;8:299308.

10. Luchetti F, Canonico B, Curci R, Battistelli M, Mannello F, Papa S, et al. Melatonin prevents apoptosis induced by UV-B treatment in U937 cell line. J Pineal Res 2006;40:158-67.

11. Luchetti F, Canonico B, Mannello F, Masoni C, D'Emilio A, Battistelli M, , et al. Melatonin reduces early changes in intramitochondrial cardiolipin during apoptosis in U937 cell line. Toxicol In Vitro 2007;21:293-301.

12. Sestili P, Barbieri E, Martinelli C, Battistelli M, Guescini M, Vallorani L, et al. Creatine supplementation prevents the inhibition of myogenic differentiation in oxidatively injured $\mathrm{C} 2 \mathrm{C} 12$ murine myoblasts. Mol Nutr Food Res 2009;53:1187-204.

13. Burattini S, Salucci S, Baldassarri V, Accorsi A, Piatti E, Madrona A, et al. Anti-apoptotic activity of hydroxytyrosol and hydroxytyrosyl laurate. Food Chem Toxicol 2013;55:248-56.

14. Salucci S, Burattini S, Battistelli M, Baldassarri V, Curzi D, Valmori A, et al. Melatonin prevents chemical-induced haemopoietic cell death. Int J Mol Sci 2014;15:6625-40.

15. Slominski A, Fischer TW, Zmijewski MA. On the role of melatonin in skin physiology and pathology. Endocrine 2005;27:137-148.

16. Tan DX, Manchester LC, Hardeland R, Lopez-Burillo S, Mayo JC, Sainz RM, et al. Melatonin: a hormone, a tissue factor, an autocoid, a paracoid, and an antioxidant vitamin. J Pineal Res 2003; 4:75-8.

17. Reiter RJ, Tan DX, Maldonado MD. Melatonin as an antioxidant: physiology versus pharmacology. J Pineal Res 2005;39:215-6.

18. Sestili P, Barbieri E, Martinelli C, Battistelli M, Guescini M, Vallorani L, et al. Creatine supplementation prevents the inhibition of myogenic differentiation in oxidatively injured $\mathrm{C} 2 \mathrm{C} 12$ 
murine myoblasts. Mol Nutr Food Res 2009;53:1187-204.

19. Sestili P, Martinelli C, Colombo E, Barbieri E, Potenza L, Sartini S, et al. Creatine as an antioxidant. Amino Acids 2011;40:138596.

20. $\mathrm{Hu} \mathrm{T}, \mathrm{He} \mathrm{XW}$, Jiang JG, Xu XL. Hydroxytyrosol and its potential therapeutic effects. J Agric Food Chem 2014;62:1449-55.

21. Franco MN, Galeano-Díaz T, López O, Fernández-Bolaños JG, FernándezBolaños JG, Sánchez J, De Miguel C, et al. Phenolic compounds and antioxidant capacity of virgin olive oil. Food Chem 2014;163:289-98.

22. Mateos R, Trujillo M, Pereira-Caro G, Madrona A, Cert A, Espartero JL. New lipophilic tyrosyl esters. Comparative antioxidant evaluation with hydroxytyrosyl esters. J Agric Food Chem 2008;56:10960-6.

23. Madrona Y, Tripathi S, Li H, Poulos TL. Crystal structures of substrate-free and nitrosyl cytochrome P450cin: implications for $\mathrm{O}(2)$ activation. Biochemistry 2014;51:6623-31.

24. Salucci S, Burattini S, Battistelli M, Buontempo F, Canonico B, Martelli AM, et al. Tyrosol prevents apoptosis in irradiated keratinocytes. J Dermatol Sci 2015;8:61-8.

25. Battistelli M, Salucci S, Olivotto E, Facchini A, Minguzzi M, Guidotti S, et al. Cell death in human articular chondrocyte: a morpho-functional study in micromass model. Apoptosis 2014;19: 1471-83.

26. Battistelli M, Salucci S, Guescini M, Curzi D, Stocchi V, Falcieri E. skeletal muscle cell behavior after physical agent treatments. Curr Pharm Des
2015;21:3665-72.

27. Salamanna F, Frizziero A, Pagani S, Giavaresi G, Curzi D, Falcieri E, et al. Metabolic and cytoprotective effects of in vivo peri-patellar hyaluronic acid injections in cultured tenocytes. Connect Tissue Res 2015;56:35-43.

28. Salucci S, Burattini S, Curzi D, Buontempo F, Martelli AM, Zappia G, et al. Antioxidants in the prevention of UVB-induced keratynocyte apoptosis. J Photochem Photobiol B 2014;41:1-9.

29. Fischer TW, Zmijewski MA, Wortsman J, Slominski A. Melatonin maintains mitochondrial membrane potential and attenuates activation of initiator (casp9) and effector caspases (casp-3/casp-7) and PARP in UVR-exposed HaCaT keratinocytes. J Pineal Res 2008;44:397407.

30. Choi YJ, Uehara Y, Park JY, Kim SJ, Kim SR, Lee HW, et al. Chng MHY884, a newly synthesized tyrosinase inhibitor, suppresses UVB-induced activation of NF- $\kappa B$ signaling pathway through the downregulation of oxidative stress. Bioorg Med Chem Lett 2014;24:1344-8.

31. Slominski A, Pisarchik A, Semak I, Sweatman T, Wortsman J, Szczesniewski A, et al. Serotoninergic and melatoninergic systems are fully expressed in human skin. FASEB J 2002;16:896-98.

32. Slominski A, Wortsman J, Tobin DJ. The cutaneous serotoninergic/melatoninergic system: securing a place under the sun. FASEB J 2005;19:17694.

33. Scheuer C, Pommergaard HC, Rosenberg J, Gögenur I. Melatonin's protective effect against UV radiation: a systematic review of clinical and experimental studies. Photodermatol Photoimmunol Photomed 2014;30:180-8. [Epub 2013 Nov 17].

34. Canonico B, Luchetti F, Ambrogini P, Arcangeletti M, Betti M, Cesarini E, et al. Pharmacological doses of melatonin induce alterations in mitochondrial mass and potential, bcl-2 levels and $\mathrm{K}+$ currents in UVB-exposed U937 cells. Cell Biol Int 2013;37:213-26.

35. Slominski AT, Zmijewski MA, Semak I, Zbytek B, Pisarchik A, Li W, et al. Cytochromes p450 and skin cancer: role of local endocrine pathways. Anticancer Agents Med Chem 2014;14:77-96.

36. Desotelle JA, Wilking MJ, Ahmad N. The circadian control of skin and cutaneous photodamage. Photochem Photobiol 2012; 88:1037-1047.

37. Srinivasan V, Pandi-Perumal SR, Brzezinski A, Zbytek B, Pisarchik A, Li $\mathrm{W}$, et al. Melatonin, immune function and cancer. Recent Pat Endocr Metab Immune Drug Discov 2011;5:109-23.

38. Hipler UC, Fischer TW, Elsner P. $\mathrm{HaCaT}$ cell proliferation influenced by melatonin. Skin Pharmacol Appl Skin Physiol 2003;16:379-85.

39. Izykowska I, Piotrowska A, PodhorskaOkołów M, Cegielski M, Zabel M, Dziegiel P. [The protective role of melatonin in the course of UV exposure].[Article in Polish]. Postepy Hig Med Dosw (Online) 2008;62:23-7.

40. Procopio A, Celia C, Nardi M, Oliverio M, Paolino D, Sindona G. Lipophilic hydroxytyrosol esters: fatty acid conjugates for potential topical administration J Nat Prod 2011;28;74:2377-81. 